\title{
Implications of laparoscopic inguinal hernia repair on open, laparoscopic, and robotic radical prostatectomy
}

\author{
Dan Spernat, David Sofield ${ }^{1}$, Daniel Moon², Mark Louie-Johnsun ${ }^{3}$, Henry H. Woo ${ }^{4}$ \\ Department of Urology, The Queen Elizabeth Hospital, Adelaide University, South Australia, Australia \\ ${ }^{1}$ Department of Urology, Fremantle Hospital, Perth, Australia \\ ${ }^{2}$ Director of Robotic Surgery, Epworth Healthcare, Victoria, Australia \\ ${ }^{3}$ Department of Urology, Gosford Private Hospital, New South Wales, Australia \\ ${ }^{4}$ Sydney Adventist Hospital Clinical School, University of Sydney, New South Wales, Australia
}

Purpose: There have been anecdotal reports of surgeons having to abandon radical prostatectomy (RP) after laparoscopic inguinal hernia repair (LIHR) due to obliteration of tissue planes by mesh. Nodal dissection may also be compromised. We prospectively collected data from four experienced prostate surgeons from separate institutions. Our objective was to evaluate the success rate of performing open RP (ORP), laparoscopic RP (LRP) and robotic assisted RP (RALRP) and pelvic lymph node dissection (PLND) after LIHR, and the frequency of complications.

Methods: A retrospective analysis of prospectively maintained databases of men who underwent RP after LIHR between 2004 and 2010 at four institutions was undertaken. The data recorded included age, preoperative prostate-specific antigen, preoperative Gleason score, and clinical stage. The operative approach, success or failure to perform RP, success or failure to perform PLND, pathological stage, and complications were also recorded.

Results: A total of 1,181 men underwent RP between 2004 and 2010. Fifty-seven patients (4.8\%) underwent RP after LIHR. An ORP was attempted in 19 patients, LRP in 33, and RALRP in 5 . All 57 cases were able to be successfully completed. Ten of the 18 open PLND were able to be completed (55.6\%). Four of the 22 laparoscopic LND were able to be completed (18.2\%). Robotic LND was possible in 5 of 5 cases (100\%). Therefore, it was not possible to complete a LND 56.8\% of patients. Complications were limited to ten patients. These complications included one LRP converted to ORP due to failure to progress, and one rectourethral fistula in a salvage procedure post failed high intensity focused ultrasound.

Conclusions: LIHR is an increasingly common method of treating inguinal hernias. LIHR is not a contra-indication to RP. However PLND may not be possible in over 50\% of patients who have had LIHR. Therefore, these patients may be under-staged and under treated.

Keywords: Prostate cancer, Inguinal hernia, Prostatectomy

\section{INTRODUCTION}

Radical prostatectomy (RP) can be a challenging operation regardless of the surgical technique employed. There have been anecdotal reports of surgeons having to abandon RP after laparoscopic inguinal hernia repair (LIHR) due to interference or obliteration of tissue planes by mesh. Moreover, nodal dissection may also be compromised by the mesh.

LIHR is less commonly performed than open inguinal hernia repair [1]. However, LIHR is becoming increasingly popular in North America and Europe [2]. The percentage of hernia repairs performed laparoscopically varies from $1 \%$ to $30 \%$ depending on the centre [2]. The number of LIHR performed is likely to increase over time as more surgeons become fa-

Corresponding author: Henry H. Woo

Sydney Adventist Hospital Clinical School, University of Sydney, 185 Fox Valley Rd Wahroonga, New South Wales 2076, Australia

E-mail: hwoo@urologist.net.au / Tel: +61294879111 / Fax: +61294738969

Submitted: 14 November 2013 / Accepted after revision: 30 December 2013 
miliar with the technique.

There is an increasing trend to manage patients with D'Amico low risk prostate cancer with active surveilance [3]. Therefore, the percentage of patients undergoing RP with D'Amico intermediate or high risk factors is increasing. These patients may benefit from pelvic lymph node dissection (PLND) [4].

We prospectively collected data from four experienced prostate surgeons from separate institutions. We report on our experience with open RP (ORP), laparoscopic RP (LRP) and robotic assisted RP (RALRP). Our objective was to evaluate the success rate of performing RP and PLND after LIHR with the three most common operative techniques.

\section{MATERIALS AND METHODS}

A retrospective analysis of prospectively maintained databases of men who underwent RP after LIHR between 2004 and 2010 at four institutions was undertaken. The operative approach and decision to perform a PLND were at the surgeons' discretion. PLND was attempted in two of 11 patients with D'Amico low risk prostate cancer and 27 of 31 patients with D'Amico Intermediate risk prostate cancer. All patients with D'Amico high risk prostate cancer (15) had attempted PLND.

The data recorded included age, preoperative prostate-specific antigen (PSA), preoperative Gleason score, and clinical stage. The operative approach, success or failure to perform $\mathrm{RP}$, pathological stage, and complications were also recorded.

The specific placement of the mesh was also recorded as left, right or bilateral. Moreover, the impact that this had on PLND was recorded including whether only unilateral PLND was possible due to interference or obliteration of tissue planes by mesh. A successful PLND was defined as a bilateral obturator lymph node (LN) dissection. However, it was noted if only a unilateral PLND was possible.

Table 1. Patient characteristics

\begin{tabular}{lccc}
\hline Characteristic & ORP & LRP & RALRP \\
\hline RP number & 19 & 33 & 5 \\
Mean age (yr) & 62.6 & 63 & 55.4 \\
Mean PSA (ng/mL) & 5.5 & 8.2 & 6.4 \\
Median Gleason grade & 7 & 7 & 7 \\
Attempted PLND & $18 / 19$ & $22 / 33$ & $5 / 5$ \\
Successful PLND, n (\%) & $10 / 18(55.6)$ & $4 / 22(18.2)$ & $5 / 5(100)$ \\
Bilateral LIHR+Successful & $3 / 7(43)$ & $3 / 12(25)$ & $3 / 3(100)$ \\
$\quad$ PLND, n (\%) & & & \\
Unilateral LIHR+Successful & $7 / 10(70)$ & $1 / 10(10)$ & $2 / 2(100)$ \\
$\quad$ Bilateral PLND, n (\%) & & & \\
\hline
\end{tabular}

$\mathrm{RP}$, radical prostatectomy; ORP, open RP; LRP, laparoscopic RP; RALRP, robotic assisted RP; PSA, prostate-specific antigen; LIHR, laparoscopic inguinal hernia repair; PLND, pelvic lymph node dissection.

\section{RESULTS}

In our multi-institution study, a total of 1,181 men underwent RP between 2004 and 2010. Fifty-seven patients (4.8\%) underwent RP after LIHR with 28 patients having a previous bilateral LIHR (2.4\%) and 29 a unilateral LIHR (2.4\%). An ORP was attempted in 19 patients, LRP in 33, and RALRP in 5 . The patients' characteristics are shown in Table 1. The mean age and PSA were 60.3 years, and $6.7 \mathrm{ng} / \mathrm{mL}$ respectively.

All $57 \mathrm{RP}$ cases were able to be successfully completed with no cases abandoned. Of the 19 ORP a PLND was attempted in 18 cases. Only 10 of the 18 open PLND were able to be completed (55.6\%). Of the 33 laparoscopic cases, PLND was attempted in 22 . Only 4 of these cases $(18.2 \%)$ were able to have a bilateral PLND and a further 9 (40.9\%) had a unilateral dissection. Robotic PLND was possible in 5 of 5 cases (100\%).

Combining data from all methods of RP, a PLND was attempted in 44 of the 57 patients (77.2\%). However, it was not possible to complete a PLND in 25 of the 44 patients (56.8\%). This was due to the proximity of the mesh to the pelvic side wall and resultant fibrotic reaction preventing clean dissection of the iliac vessels and Obturator nerve.

Complications were limited to ten patients. These complications are presented in Table 2. Complications were graded using the Clavien-Dindo [5] system. There were two grade 3a complications which included a lymph leak and an urinoma. Both of these collections required radiological drainage and settled without further sequelae.

There were 4 grade $3 \mathrm{~b}$ complications. Two of these complications were recognised and corrected at the time of RP. These included the conversion of a LRP to an ORP due to failure to progress, and an inadvertent cystotomy. The recto urethral fistulae occurred in a patient who had an ORP post failed high

Table 2. Complications of RP post-LIHR

\begin{tabular}{lcc}
\hline Complication & No. & $\begin{array}{c}\text { Clavien-Dindo } \\
\text { classification }\end{array}$ \\
\hline AUR & 1 & 2 \\
Wound infection & 1 & 2 \\
Blood transfusion & 2 & 2 \\
Lymph leak & 1 & $3 \mathrm{a}$ \\
Urinoma & 1 & $3 \mathrm{a}$ \\
LRP converted to ORP & 1 & $3 \mathrm{~b}$ \\
Recto-urethral fistulae (salvage HIFU) & 1 & $3 \mathrm{~b}$ \\
Cystotomy & 1 & $3 \mathrm{~b}$ \\
Bladder neck contracture & 1 & $3 \mathrm{~b}$ \\
Total & 10 & \\
\hline
\end{tabular}

$\mathrm{RP}$, radical prostatectomy; LIHR, laparoscopic inguinal hernia repair; AUR, acute urinary retention; LRP, laparoscopic RP; ORP, open RP; HIFU, high intensity focused ultrasound. 
intensity focused ultrasound. This was discovered in the post operative period and required a defunctioning colostomy. The colostomy was able to be reversed and the patient recovered well. The bladder neck contracture required a bladder neck incision and was able to be stabilised endoscopically.

\section{DISCUSSION}

Prior LIHR has a risk of abandonment of RP due to the loss of normal tissue planes. Subsequent reports have shown that LIHR is not a contra-indication to ORP [6]. However, this is the first paper that evaluates the safety of ORP, LRP, and RALRP after LIHR. In our series all 57 patients had a successful RP post-LIHR. Thus RP is a safe and reasonable treatment option for patients with prostate cancer. The laparoscopically placed mesh does make ORP, LRP and RALRP more difficult.

We recognize the limitations of our study. This study is limited by the small numbers of patients involved, particularly in the RALRP group. Additionally, all data was retrospectively reviewed from a prospective database. While this study demonstrates that RP is safe post LIHR, we omitted to document the impact that LIHR has on the RP surgical time. Moreover, our study would have been enhanced by greater homogeneity of surgical technique and management.

Additional information from our general surgical colleagues may have allowed us to determine good and poor prognostic factors for RP and PLND post LIHR. These factors include the size of the mesh used, whether the LIHR was performed transperitoneally or extraperitoneally, the method of mesh attachment and the duration of time between LIHR and RP.

Regardless of RP technique the mesh and resultant fibrosis impede retraction. Furthermore, the mesh may be adherent to the bladder. This requires careful dissection to prevent inadvertent cytotomies. The advantage with LRP and RALRP is that it is not necessary to cut through the mesh to gain access to the retropubic space. However, it is necessary to mobilise the bladder from the surrounding fibrosis to allow the bladder to parachute down for the urethral anastomosis.

The placement of mesh for a LIHR may not be confined to the affected side. Depending on the approach taken by the general surgeon involved, the mesh may be placed across the midline and even cover the contralateral External Iliac Vein. This may prevent the safe dissection of the contralateral obturator LNs. Moreover, there are reports in the literature of mesh migrating from its original position leading to significant morbidity [7]. As a result it is recommended that when mesh is placed pre-peritoneally that all tears in the peritoneum are repaired, and it is secured to surrounding structures [8].
PLND offers important prognostic information and patients with limited LN metastases may derive a therapeutic benefit. Moreover, PLND is the most accurate staging procedure for the presence of LN metastases [9]. There is debate in the literature as to who would benefit from PLND and the extent of the PLND. The 2010 National Comprehensive Cancer Network guidelines state that any patient with a $2 \%$ risk of $\mathrm{LN}$ metastases should undergo PLND [10]. Meanwhile the current European Association of Urology guidelines are more moderate and recommend that patients with higher than $7 \%$ risk of $\mathrm{LN}$ metastases undergo PLND [4]. It is estimated that patients with D'Amico low risk prostate cancer have a risk of LN metastases of less than 7\% [10]. However, the risk of LN metastases in patients with a Gleason score $\geq 7$ is $25 \%$ [11].

Touijer et al. [12] have demonstrated that by including the hypogastric, external iliac and obturator fossa LNs (ePLND) there is a statistically significant increased rate of positive LN detected compared to external iliac node dissection alone. ePLND has shown a higher detection of nodal metastases (14.3\% vs. $4.5 \%, P=0.004$ ) [12]. Moreover, this dissection also resulted in a statistically significant increase in the median number of nodes resected (13 vs. 9 , respectively, $P<0.001$ ) [12].

However, the patients undergoing ePLND had a significantly longer operating time (30 minutes, $P<0.001)$ [11]. In addition, compared to patients omitting PLND, there was a statistically significant increase in postoperative complications $(P<0.001)[12]$. The data revealed in Touijer's populaton, if 1,000 patients underwent ePLND compared to no PLND, an additional 140 patients would have nodal metastases detected at the cost of an additional 40 patients experiencing a grade 3 complication [12]. Likewise, for every 10 patients who only underwent an external iliac LN dissection one patient with LN metastases were missed compared with those patients who underwent ePLND [12].

In addition to its diagnostic role, some authors suggest that PLND may also have a therapeutic role [13]. The National Cancer Institute Surveillance Epidemiology and End Results Program database followed patients undergoing RP for greater than 10 years. This database demonstrated the larger the number of nodes dissected the greater the likelihood of identifying LN metastasis and improved prostate cancer specific survival [14]. This benefit is also conveyed to node-negative patients raising speculation that adequate PLND may control occult micro-metastatic nodal disease [14].

Thus all patients with a risk of LN metastases in excess of $7 \%$ should undergo bilateral PLND. However this may not be possible in up to $56.8 \%$ of patients who have had LIHR. In our series only $43.2 \%$ of PLND after LIHR were possible. The most 
success was encountered with RALRP, however as this group only has 5 patients, it is difficult to draw any conclusions. The least success was found with the LRP group, with only $18.2 \%$ of patients being able to undergo a successful PLND. Thus after LIHR there are a large percentage of patients who may be under treated and under staged.

In conclusion, LIHR is an increasingly common method of treating inguinal hernias. LIHR is not a contra-indication to RP. However PLND may not be possible in over $50 \%$ of patients who have had LIHR. Therefore, these patients may be under-staged and under treated.

\section{CONFLICT OF INTEREST}

No potential conflict of interest relevant to this article was reported.

\section{REFERENCES}

1. Takata MC, Duh QY. Laparoscopic inguinal hernia repair. Surg Clin North Am 2008;88:157-78.

2. Kulacoglu H. Current options in inguinal hernia repair in adult patients. Hippokratia 2011;15:223-31.

3. Ischia JJ, Pang CY, Tay YK, Suen CF, Aw HC, Frydenberg M. Active surveillance for prostate cancer: an Australian experience. BJU Int 2012;109 Suppl 3:40-3.

4. Heidenreich A, Bellmunt J, Bolla M, Joniau S, Mason M, Matveev V, et al. EAU guidelines on prostate cancer. Part 1: screening, diagnosis, and treatment of clinically localised disease. Eur Urol 2011;59:61-71.

5. Dindo D, Demartines N, Clavien PA. Classification of surgical complications: a new proposal with evaluation in a cohort of 6336 patients and results of a survey. Ann Surg 2004;240:205-13.
6. Saint-Elie DT, Marshall FF. Impact of laparoscopic inguinal hernia repair mesh on open radical retropubic prostatectomy. Urology 2010;76:1078-82.

7. Yilmaz I, Karakas DO, Sucullu I, Ozdemir Y, Yucel E. A rare cause of mechanical bowel obstruction: mesh migration. Hernia 2013;17:267-9.

8. Lo DJ, Bilimoria KY, Pugh CM. Bowel complications after prolene hernia system (PHS) repair: a case report and review of the literature. Hernia 2008;12:437-40.

9. Briganti A, Blute ML, Eastham JH, Graefen M, Heidenreich A, Karnes JR, et al. Pelvic lymph node dissection in prostate cancer. Eur Urol 2009;55:1251-65.

10. Briganti A, Chun FK, Salonia A, Gallina A, Farina E, Da Pozzo LF, et al. Validation of a nomogram predicting the probability of lymph node invasion based on the extent of pelvic lymphadenectomy in patients with clinically localized prostate cancer. BJU Int 2006;98:788-93.

11. Pound CR, Partin AW, Eisenberger MA, Chan DW, Pearson JD, Walsh PC. Natural history of progression after PSA elevation following radical prostatectomy. JAMA 1999;281:1591-7.

12. Touijer K, Fuenzalida RP, Rabbani F, Paparel P, Nogueira L, Cronin AM, et al. Extending the indications and anatomical limits of pelvic lymph node dissection for prostate cancer: improved staging or increased morbidity? BJU Int 2011;108: 372-7.

13. Masterson TA, Bianco FJ Jr, Vickers AJ, DiBlasio CJ, Fearn PA, Rabbani F, et al. The association between total and positive lymph node counts, and disease progression in clinically localized prostate cancer. J Urol 2006;175:1320-4.

14. Godoy G, Chong KT, Cronin A, Vickers A, Laudone V, Touijer $K$, et al. Extent of pelvic lymph node dissection and the impact of standard template dissection on nomogram prediction of lymph node involvement. Eur Urol 2011;60:195-201. 